\title{
Stochastic excitation of gravity modes in massive main-sequence stars
}

\author{
R. Samadi · K. Belkacem • M.J. Goupil • M.-A. Dupret • \\ A.S. Brun • A. Noels
}

Received: 28 September 2009 / Accepted: 17 November 2009 / Published online: 1 December 2009

(C) Springer Science+Business Media B.V. 2009

\begin{abstract}
We investigate the possibility that gravity modes can be stochastically excited by turbulent convection in massive main-sequence (MS) stars. We build stellar models of MS stars with masses $M=10 M_{\odot}, 15 M_{\odot}$, and $20 M_{\odot}$. For each model, we then compute the power supplied to the modes by turbulent eddies in the convective core (CC) and the outer convective zones (OCZ). We found that, for asymptotic gravity modes, the major part of the driving occurs within the outer iron convective zone, while the excitation of low $n$ order modes mainly occurs within the CC. We compute the mode lifetimes and deduce the expected mode amplitudes. We finally discuss the possibility of detecting such stochastically-excited gravity modes with the CoRoT spacebased mission.
\end{abstract}

Keywords Turbulence Convection - Oscillations · Mode driving $\cdot$ Massive stars

\section{Introduction}

Stochastic excitation of solar gravity modes by turbulent convection has been recently studied in detail by Belkacem

R. Samadi ( $\varangle) \cdot$ M.J. Goupil

Observatoire de Paris, LESIA, CNRS UMR 8109,

92195 Meudon, France

e-mail: reza.samadi@obspm.fr

K. Belkacem · M.-A. Dupret · A. Noels

Institut d'Astrophysique et de Géophysique, Université de Liège, Allée du 6 Août 17-B, 4000 Liège, Belgium

A.S. Brun

DSM/DAPNIA/SAp, CEA Saclay, 91191 Gif-sur-Yvette Cedex,

France et al. (2009b). The modelling performed by these authors put the theoretical amplitudes of solar gravity modes close to the SoHO/GOLF detection threshold. A new issue then arises: can we expect such stochastically-excited gravity modes to be more easily detectable in other stars than the Sun?

Main-sequence stars significantly more massive than the Sun are potentially interesting for seeking stochasticallyexcited modes. Indeed, stochastically-excited $p$ modes have recently been detected in a massive star (Belkacem et al. 2009a). The question remains: can gravity modes be stochastically excited in such stars? Stars with mass above $M \approx 1.2 M_{\odot}$ have a convective core (CC hereafter) and the more massive the star, the higher is the eddies' kinetic energy in the $\mathrm{CC}$. We therefore consider here the illustrative cases of massive main-sequence stars with masses $M=$ $10 M_{\odot}, 15 M_{\odot}$, and $20 M_{\odot}$. Amplitudes of stochasticallyexcited gravity modes are computed following Belkacem et al. (2009b) as explained in Sect. 2. We describe in Sect. 3 the efficiency of the different driving regions. The results of our calculations are presented and commented on in Sect. 4, and our conclusions are summarized in Sect. 5. We finally discuss in Sect. 5 the possibility of detecting stochasticallyexcited gravity modes with the CoRoT space-based mission.

\section{Mode amplitudes}

We compute the mean-squared surface velocity for each non-radial mode according to (see e.g. Belkacem et al. 2009b, and references therein):

$v_{\mathrm{s}}^{2}=\frac{\mathcal{P}}{2 \eta \mathcal{M}}$ 
where $\mathcal{P}$ is the mode excitation rate, $\eta$ the mode damping rate (which is equal to the inverse of the mode lifetime $\tau$ ) and $\mathcal{M}$ the mode mass. The latter is defined as

$\mathcal{M}=\frac{I}{\xi_{\mathrm{r}}^{2}\left(R_{*}\right)+\ell(\ell+1) \xi_{\mathrm{h}}^{2}\left(R_{*}\right)}$

where $I$ is the mode inertia, $\ell$ the mode angular degree, $\xi_{\text {r }}$ and $\xi_{\mathrm{h}}$ respectively are the radial and the horizontal components of the mode eigendisplacement. Both quantities are evaluated here at the photosphere, i.e. at the radius $r=R_{*}$ where $R_{*}$ is the stellar radius. The mode inertia is defined as $I=\int \mathrm{d} m\left(\xi_{\mathrm{r}}^{2}(r)+\ell(\ell+1) \xi_{\mathrm{h}}^{2}(r)\right)$.

The mode amplitude in terms of the intensity (or brightness fluctuations) is then deduced at the photosphere according to (Dziemblowski 1977; Pesnell 1990)

$\frac{\delta L}{L}=4 \frac{\delta T_{\mathrm{eff}}}{T_{\mathrm{eff}}}+2 \frac{\delta R_{*}}{R_{*}}$

where $\delta L$ is the bolometric intensity fluctuation, $\delta T_{\text {eff }}$ the effective temperature fluctuation, and $\delta R_{*}$ the variation of the stellar radius for each given mode. As seen in (1), the modal amplitude is a balance between driving $(\mathcal{P})$ and damping $(\eta)$. Determination of the mode amplitude then requires knowledge of $\mathcal{P}$ (see Sect. 2.1) and $\eta$ (see Sect 2.2).

\subsection{Excitation rates $(\mathcal{P})$}

Mode excitation rates are computed on the basis of the formalism due to Belkacem et al. (2008). This formalism is a generalization of the work of Samadi and Goupil (2001, SG01 hereafter) to non-radial modes. Two sources of driving are taken into account: the Reynolds stress tensor and the advection of the turbulent fluctuations of entropy by the turbulent motions (the "entropy source term").

In the $\mathrm{CC}$, the entropy contribution represents less than $\sim 10 \%$ of the total excitation rates. This is because the gravity modes are evanescent within the CC. Accordingly, the second derivative of displacement eigenfunction is negligible and so is the entropy source term (see details in Belkacem et al. 2008, 2009b). On the other hand, in the outer convective zone (OCZ hereafter), this source term is not negligible since it represents up to $\sim 50 \%$. This is a consequence of the fact that energy is inefficiently transported by convection within the OCZ. As a consequence, non-adiabatic fluctuations of the gas pressure are important compared to the turbulent pressure. For the sake of brevity we will not discuss further the entropy contribution.

When limited to Reynolds stresses, the excitation rates, $\mathcal{P}$, can be written as (see (21) of Belkacem et al. 2008)

$$
\begin{aligned}
\mathcal{P}= & \frac{\pi^{3}}{2 I} \int_{0}^{M} \mathrm{~d} m \frac{\rho_{0} u_{0}^{4}}{k_{0}^{3} \omega_{0}} \mathcal{R}(\vec{r}, m) \mathcal{S}_{\mathrm{R}}\left(\omega_{\mathrm{osc}}, m\right) \\
\mathcal{S}_{\mathrm{R}}= & \frac{k_{0}^{3} \omega_{0}}{u_{0}^{4}} \int_{0}^{+\infty} \frac{\mathrm{d} k}{k^{2}} E^{2}(k) \\
& \times \int_{-\infty}^{+\infty} \mathrm{d} \omega \chi_{k}\left(\omega+\omega_{\mathrm{osc}}\right) \chi_{k}(\omega)
\end{aligned}
$$

where $m$ is the local mass, $\rho_{0}$ the mean density, $\omega_{\text {osc }}$ the mode angular frequency, $u_{0}$ a characteristic velocity associated with the energy bearing eddies, $\mathcal{S}_{\mathrm{R}}$ the dimensionless source function associated with the Reynolds stress, $E(k)$ the spatial kinetic energy spectrum, $\chi_{k}$ the eddy-time correlation function, and $k$ the wave number. The term $\mathcal{R}(r)$ depends on the eigenfunction, its expression is given in (23) of Belkacem et al. (2008). We have introduced, for convenience, the characteristic frequency $\omega_{0} \equiv k_{0} u_{0}$ and the characteristic wave number $k_{0} \equiv 2 \pi / \Lambda$ where $\Lambda$ is the characteristic size of the energy bearing eddies.

\subsection{Damping rates $(\eta)$}

Mode damping rates $(\eta)$ are computed using the full nonadiabatic and non-radial pulsation code MAD (Dupret 2002; Dupret et al. 2003). This code includes a time-dependent convection (TDC) treatment described in Grigahcène et al. (2005), which allows us to take into account the role played by the variations of the convective flux, the turbulent pressure, and the dissipation rate of the turbulent kinetic energy. Actually, the damping of the gravity modes is dominated by the perturbation of the radiative flux. We have numerically verified that the interaction between convection and pulsation does not affect the damping rates.

\subsection{Computational details and assumptions}

We consider main-sequence stellar models computed with the stellar evolution code CLÉS (Scuflaire et al. 2008). These models use standard physics, in particular convection is treated according to the Böhm-Vitense mixing-length (MLT) formalism, but overshoot is not included. Details of the physical inputs used are given in (Belkacem et al. 2009c). Three models are computed; one with a mass $M=$ $10 M_{\odot}\left(\right.$ M10 hereafter), the second with $M=15 M_{\odot}(\mathrm{M} 15$ hereafter), and the third with $M=20 M_{\odot}$ (M20 hereafter). All models are at the same evolutionary stage, namely $X_{\mathrm{c}}=$ 0.5 where $X_{\mathrm{c}}$ is the hydrogen core abundance.

The mode damping rates $(\eta)$ or equivalently the mode lifetimes $(\tau=1 / \eta)$, as well as the relation between $\delta T_{\text {eff }}$ and $\delta R_{*}$ are obtained using the pulsation code MAD (see Sect. 2.2).

The rate $(\mathcal{P})$ at which energy is injected into a mode per unit time is then computed according to (4). The density 
stratification, $\rho_{0}$, is obtained from the equilibrium model. Mode eigenfrequencies and eigenfunctions are computed using the adiabatic pulsation code OSC (Scuflaire et al. 2008). We consider modes with angular degree $\ell=1$ and radial orders between $n=-2$ and $n=-21$ (asymptotic $\mathrm{g}$ mode). Modes with $\ell>1$ are not considered, since those modes are expected to have lower amplitudes. Modes below $n=-21$ are not considered for several reasons: the calculation of gravity modes with lower values of $n$ requires models with a much denser mesh-grid than those at our disposal. Furthermore, in the limit where $n \rightarrow-\infty$, the density of gravity modes becomes very high in frequency. Below a given value of $n$, it is then no longer possible to distinguish in the Fourier spectrum one mode from another.

Apart from the eigenfunctions and $\rho_{0}$, (4) involves both the eddy-time correlation function $\left(\chi_{k}\right)$ and the turbulent kinetic energy spectrum $(E)$. However, the properties of turbulent convection are poorly known for main-sequence massive stars, in particular in the CC (see the discussion in Sect. 5). We therefore need to set a priori $\chi_{k}$, the $k$ dependence of $E$ and the characteristic wave number $\left(k_{0}\right)$ or equivalently the characteristic length scale $(\Lambda)$ at which energy is injected into the turbulent cascade.

- $\chi_{k}$ : It has been shown that the use of a Lorentzian function for $\chi_{k}$ results for the Sun (Samadi et al. 2003; Belkacem et al. 2006) and $\alpha$ Cen A (Samadi et al. 2008) in good agreement between observed mode amplitudes and computed ones. We will then by default consider a Lorentzian for $\chi_{k}$. However, in order to probe the sensitivity of our results to this choice we will also consider for comparison a Gaussian for $\chi_{k}$ (see Sect. 5).

- $E(k)$ : Among the different analytical functions tested so far for $E$ (see e.g. Samadi et al. 2003; Chaplin et al. 2005), the best agreement with a solar 3D simulation was found with the so-called "Extended Kolmogorov Spectrum" defined by Musielak et al. (1994). We will then use by default this analytical empirical spectrum.

- $\Lambda$ : One usually assumes by default that $\Lambda$ is proportional to the classical mixing length ( $\left.\Lambda_{\mathrm{MLT}}\right)$, i.e. $\Lambda=\beta \Lambda_{\mathrm{MLT}}$ where $\Lambda_{\mathrm{MLT}}$ is the mixing length, and $\beta$ is a free parameter introduced in order to probe the sensitive of our results to this definition of $\Lambda$ (see SG01). According to Samadi et al. (2003), one must assume $\beta=5$ in order that - in the case of the Sun- $\Lambda_{\mathrm{MLT}}$ matches the characteristic length $\Lambda$ derived from the numerical simulations of the upper part of the solar convection zone. However, in the CC, $\Lambda_{\mathrm{MLT}}$ and hence $\Lambda$ exceeds the size of the CC $\left(\Lambda_{\mathrm{c}}\right)$. In the $\mathrm{CC}$, we will therefore assume the upper limit $\Lambda=\Lambda_{\mathrm{c}}$. In order to probe our sensitivity to $\Lambda$, other choices will be investigated in Sect. 5 .

\section{Efficiency of the driving regions}

At a given layer, the power supplied to the modes by the Reynolds stress is proportional-per unit mass-to $F_{\text {kin }} \Lambda^{4} S_{\mathrm{R}} \mathcal{R}(\vec{\xi}, m) / I$ where $F_{\text {kin }}$ is the vertical flux of kinetic energy, which is proportional to $\rho_{0} u_{0}^{3}$ (for details see Samadi 2010). It is important to stress that the value taken by the dimensionless source function, $S_{R}$, depends on the way mode and turbulent eddies are time-correlated: $S_{\mathrm{R}}$ is maximum for $p_{\text {osc }} \gtrsim \tau_{0}$ (resonance) where $\tau_{0} \equiv 2 \pi / \omega_{0}$ is the characteristic eddy turn-over time and $p_{\mathrm{osc}}=2 \pi / \omega_{\mathrm{osc}}$ is the period of the mode. On the other hand $S_{\mathrm{R}}$ decreases very rapidly for $p_{\text {osc }}<\tau_{0}$ (off-resonance). $F_{\text {kin }}, \Lambda$ and $S_{\mathrm{R}}$ depend directly on the properties of the convective regions. The efficiency of the driving will then depend on the balance between these three terms. We discuss below the efficiency of the different convective regions.

We distinguish three convective regions.

- The convective zone associated with the helium opacity bump: this region is located at $T \approx 40000 \mathrm{~K}$, and is inefficient to transport energy with a negligible ratio of the convective heat flux to the radiative one. This region is located near the star surface, where density and, as a consequence, $F_{\mathrm{kin}}$ are low. The mixing length, $\Lambda_{\mathrm{MLT}}$, is more than hundred times smaller than the size of the $\mathrm{CC}\left(\Lambda_{\mathrm{c}}\right)$. Furthermore, the characteristic eddy turn-over time $\left(\tau_{0}\right)$ is very long compared to the period ( $p_{\text {osc }}$ ) of the modes we are interested in here. Driving of the gravity modes is then expected to be negligible in the helium CZ.

- The convective zone associated with the iron opacity bump, located at $T \approx 200000 \mathrm{~K}$ : as for the helium convective region, the transport of energy by convection is still inefficient. However, this region is located deeper than the helium one, where density is higher. $F_{\text {kin }}$ is still negligible compared to the total flux, but the ratio of the kinetic energy flux of the iron convective zone to the helium one is $\sim 10^{8}$ for e.g. M10. Furthermore, $\tau_{0}$ is of the order of $p_{\text {osc }}$. The excitation in this region is then expected to be much stronger than in the helium CZ.

- The convective core (CC): convection is fully efficient, meaning that most of the flux is transported by convection. The kinetic energy is several magnitudes higher than for the iron convective zone (ICZ hereafter), due to the high densities. For instance in M10, $F_{\text {kin }}$ is about $10^{5}$ larger than in the ICZ. Furthermore, $\Lambda$ is several magnitudes higher than in the ICZ. The driving is then expected to be potentially stronger in the $\mathrm{CC}$ than in the OCZ. However, $\tau_{0}$ is significantly longer than $p_{\text {osc }}$. In other words, excitation lies off-resonance within the CC. In this region, the final efficiency of the driving will depend on the net balance between $F_{\text {kin }}, S_{R}$, and $\Lambda$. 

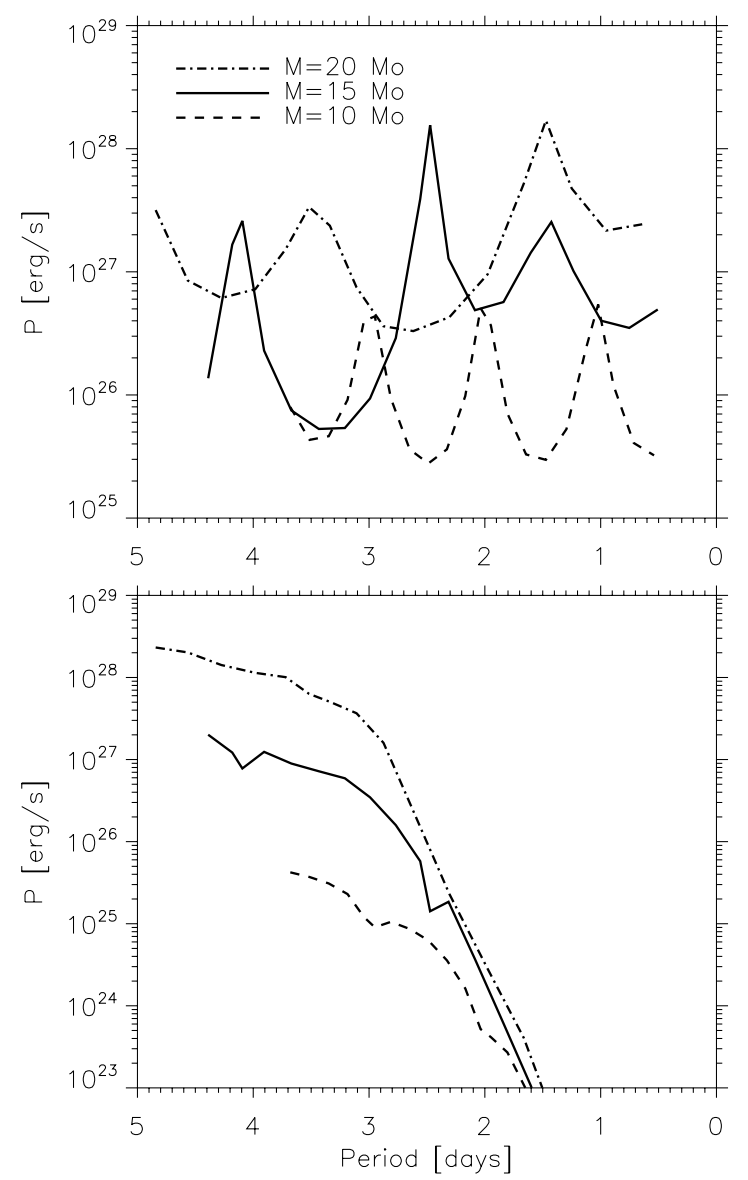

Fig. 1 Top: contribution of the $\mathrm{CC}$ to the mode excitation rates $\left(\mathcal{P}_{\mathrm{C}}\right)$ as a function of the mode period $\left(p_{\text {osc }}\right) . \mathcal{P}_{\mathrm{C}}$ is shown for the three models: $M=10 M_{\odot}$ (dashed line), $M=15 M_{\odot}$ (solid line) and $M=20 M_{\odot}$ (dot-dashed line). Bottom: contribution of the outer convective zones (OCZ) to the mode excitation rates $\left(\mathcal{P}_{\mathrm{S}}\right)$ as a function of the mode period $\left(p_{\text {osc }}\right)$. The line style is the same as in the top panel.

\section{Result}

\subsection{Excitation in the convective core}

The contribution of the $\mathrm{CC}$ to the mode excitation rates $\left(\mathcal{P}_{\mathrm{C}}\right.$ hereafter) are presented in Fig. 1 (top) for the three models. Important wiggles are seen on $\mathcal{P}_{\mathrm{C}}$. They are due to the behavior of the mode eigendisplacements in the vicinity of the CC. More precisely, mode amplitude in the $\mathrm{CC}$ depends on the phase of the eigenfunction at the frontier of the $\mathrm{CC}$.

The values reached by $\mathcal{P}_{\mathrm{C}}$ are larger for M15 compared to $\mathrm{M} 10$. On the other hand, the values reached by $\mathcal{P}_{\mathrm{C}}$ for M15 are as high as for M20. These results are explained as follows: the larger $F_{\text {kin }}$, the stronger the driving. However, $\mathcal{P}$ is inversely proportional to $I$ (see 4$)$. Hence-at fixed $F_{\text {kin }}$ - the larger $I$, the lower is $\mathcal{P}$. Generally, the larger $M$, the higher is $I$. On the other hand, the larger $M$, the higher is $F_{\text {kin }}$ in the CC. There is then a balance between $F_{\text {kin }}$ and $I$.

\subsection{Excitation in the outer convective zones}

The contribution of the outer convective zones (OCZ) to the mode excitation rates $\left(\mathcal{P}_{\mathrm{S}}\right.$ hereafter $)$ are presented in Fig. 1 (bottom). We find that, within the OCZ, the excitation predominantly occurs within the iron convective zone. This is a direct consequence of the arguments developed in Sect. 3.

As clearly seen in Fig. 1 (bottom), the larger $M$ the higher $\mathcal{P}_{\mathrm{S}}$. This is because the larger $M$, the higher the temperature at the surface. Now, the higher the temperature, the larger the energy transported by convection and accordingly the larger $F_{\text {kin. }}$.

At low frequency ( $p_{\text {osc }} \gtrsim 3$ days), $\mathcal{P}_{\mathrm{S}}$ is in general larger than $\mathcal{P}_{\mathrm{C}}$. On the other hand, this contribution decreases more rapidly with decreasing $p_{\mathrm{osc}}$ than does $\mathcal{P}_{\mathrm{C}}$. This is because $\mathcal{P}_{\mathrm{S}}$ depends on the mode compressibility, which decreases with increasing frequency (i.e. decreasing $n$ ), while $\mathcal{P}_{\mathrm{C}}$ depends on the amplitude of the mode displacement in the vicinity of the $\mathrm{CC}$, which increases with increasing frequency. At high frequency ( $p_{\text {osc }} \lesssim 3$ days), the excitation turns out to be dominated by the contribution of the $\mathrm{CC}$ $\left(\mathcal{P}_{\mathrm{C}}\right)$.

\subsection{Mode amplitudes}

We sum the contribution of the $\mathrm{CC}\left(\mathcal{P}_{\mathrm{C}}\right)$ with that of the OCZ $\left(\mathcal{P}_{\mathrm{S}}\right)$; this gives the total mode excitation rates $\mathcal{P}=$ $\mathcal{P}_{\mathrm{S}}+\mathcal{P}_{\mathrm{C}}$. From $\mathcal{P}$ and $\eta$, we then derive the mode amplitudes in terms of surface velocity $\left(v_{\mathrm{s}},(1)\right)$ and intensity $(\delta L / L,(3))$. The result is shown in Fig. 2. We have not taken the mode visibility factor into account; in other words, the amplitudes shown in Fig. 2 correspond to intrinsic (in opposition to apparent) rms amplitudes.

\section{Conclusion and discussion}

We have computed, for three main-sequence stellar models, the power $(\mathcal{P})$ supplied by turbulent convection into gravity modes. According to our theoretical calculations and associated assumptions, gravity modes are expected to be efficiently excited both in the $\mathrm{CC}$ and the iron convective zone. On the other hand, the driving within the helium $\mathrm{CZ}$ is found to be negligible compared to the driving taking place in the other driving regions.

We have next computed the mode damping rates (equivalently, the mode lifetimes). We found that the mode damping is dominated by the perturbation of the radiative flux. The computed $\eta$ are then more reliable than if the coupling between pulsation and convection were at the origin of the damping.

We have finally derived the mode amplitudes. The amplitudes of asymptotic g-modes are mainly fixed by the driving 

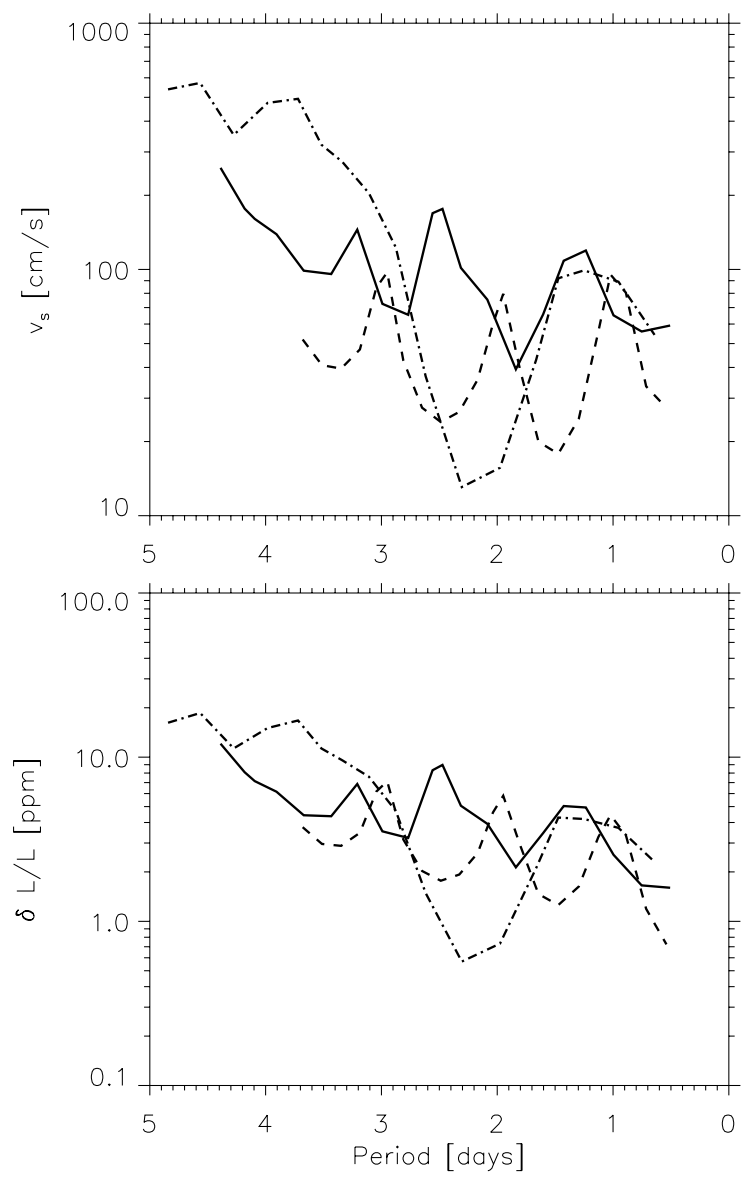

Fig. 2 Intrinsic mode amplitudes as a function of $p_{\text {osc }}$. The line style is the same as in Fig. 1. Top: mode amplitudes in terms of surface velocity, $v_{\mathrm{s}}$ (see (1)). Bottom: mode amplitudes in terms of intensity, $\delta L / L$ (see (3)).

taking place within the iron convective zone. On the other hand, the amplitudes of the low $n$ order modes are expected to be predominantly fixed by the driving within the CC. However, the relative contribution of the $\mathrm{CC}$ and the iron convective zone to the mode excitation rates significantly depends on the assumptions concerning the characteristic length $\Lambda$ and the eddy-time correlation function $\chi_{k}$.

The theoretical mode amplitudes in surface velocity $\left(v_{\mathrm{s}}\right)$ are found in general to be significantly larger than those of the solar p-modes $(\sim 30 \mathrm{~cm} / \mathrm{s})$. Mode amplitudes in intensity are found to be of the same order as for the solar p-modes ( $\simeq 2.5$ ppm, see Michel et al. 2009).

For asymptotic g-modes, the larger $M$, the larger are the mode amplitudes. This is because the higher $M$, the higher is the energy transported by convection and hence the stronger the driving within the OCZ. For low $n$ order modes, the maximum of the mode amplitudes depends weakly on $M$. Indeed, the square of the mode amplitude is inversely proportional to the mode inertia $(I)$. Furthermore, the driving within the $\mathrm{CC}$ results from the balance between the kinetic flux $\left(F_{\text {kin }}\right)$ in the $\mathrm{CC}$ and $I . F_{\text {kin }}$ in the CC increases with

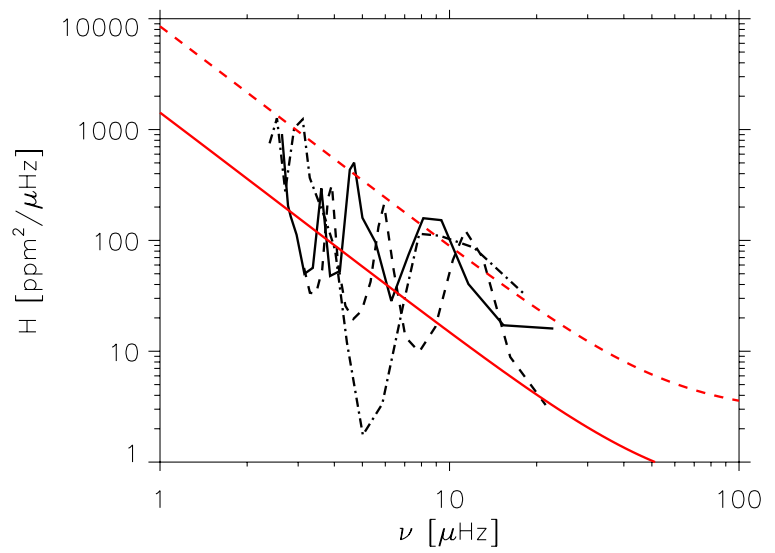

Fig. 3 Mode heights in terms of intensity and as a function of the period. The black curves have the same meaning as in Fig. 1. The red curve corresponds to an estimate of the upper limit of the CoRoT instrumental noise level (see text). The dashed red curve corresponds to the associated detection threshold (see text)

increasing $M$. For low $n$ order modes, $I$ also increases with increasing $M$. Therefore, the increase of $F_{\text {kin }}$ with $M$ is partially compensated by the increase of $I$ with $M$.

At this point several issues arise.

(i) How reliable are our amplitude estimates?

(ii) Can we detect such stochastically-excited gravity modes with CoRoT?

We now address these two questions.

Uncertainties: Among the different assumptions adopted in the present model concerning the properties of turbulent convection, the major sources of uncertainty are the characteristic length $\Lambda$, the adopted prescription for $\chi_{k}$, and the wave-number dependence of $E(k)$.

Concerning $\Lambda$, in the absence of other physical consideration, it is usually assumed-by default-that $\Lambda$ is proportional to the mixing length (which is by definition proportional to the pressure scale height). The coefficient of proportionality, $\beta$, was - as in the case of the Sun - set to 5 (see Sect. 2.3). However, in the CC, $\Lambda$ cannot be larger than $\Lambda_{\mathrm{c}}$. Therefore, we have assumed by default $\Lambda=\Lambda_{\mathrm{c}}$ in the CC. Accordingly, our theoretical calculation of the excitation in the $\mathrm{CC}$ then corresponds to an upper limit. Assuming $\Lambda=\Lambda_{\mathrm{c}} / 10$ in the $\mathrm{CC}$ results in significantly lower mode amplitudes. Concerning the OCZ, assuming as in the Sun $\beta=5$ is rather arbitrary. In order to probe the sensitivity of our results to this choice we performed a calculation for which $\Lambda=\Lambda_{\mathrm{MLT}}$ in the $\mathrm{OCZ}$ and $\Lambda=\Lambda_{\mathrm{c}} / 10$ in the CC. In that case the computed mode amplitudes are found to be about 20 times lower compared to our default case.

Concerning $\chi_{k}$ and $E(k)$, we have chosen the analytical forms that match hydrodynamical 3D simulations of the outer layer of stars analogues to the Sun and that result-in 
the case of the Sun and $\alpha$ Cen A-in good agreement between computed and observed $\mathcal{P}$. However, whether these analytical forms remain valid in the present case is an open question.

Finally, we point out that some 3D hydrodynamical models of stars with CC have been performed (see e.g. Browning et al. 2004; Meakin and Arnett 2007). These 3D models can provide some hints about the properties of turbulent convection in the CC. This will be investigated in future work.

Detection: The noise level of the CoRoT instrument in the Fourier domain can be estimated by considering the Fourier spectrum of a CoRoT star that is almost constant. We thus consider the CoRoT target HD $50170(\mathrm{~F} 2, \mathrm{mv}=6.82)$, which almost does not show intrinsic variability. We have fitted the power spectrum of this target using a Lorentzian function. The result is shown in Fig. 3. This fitted spectrum then represents an estimate of the upper limit of the CoRoT instrumental noise. From this limit we can then derive a detection threshold for a given confidence level. This threshold is presented in Fig. 3 for a confidence level of $95 \%$. This threshold must be compared with the mode height and not with the mode amplitude (see the definition of the mode height in e.g. Lochard et al. 2005). This comparison is shown in Fig. 3. The mode heights are all found below the CoRoT detection threshold associated with individual modes. We point out that taking into account the mode visibility factor would result in lower mode height. As a conclusion, the detection of stochastically-excited gravity modes with CoRoT seems to be challenging. However, an open question is whether or not the current Kepler mission or the future PLATO mission will be able to detect such modes.

Acknowledgements This work was supported by the Centre National d'Etudes Spatiales (CNES). It is based on observations with CoRoT. K.B. acknowledges financial support through a postdoctoral fellowship from the "Subside fédéral pour la recherche 2009", University of Liège. A.N is grateful to HELAS for financial support.

\section{References}

Belkacem, K., Samadi, R., Goupil, M.J., Kupka, F., Baudin, F.: Astron. Astrophys. 460, 183 (2006)

Belkacem, K., Samadi, R., Goupil, M.J., Dupret, M.A.: Astron. Astrophys. 478, 163 (2008)

Belkacem, K., Samadi, R., Goupil, M.J., Lefèvre, L., Baudin, F., Deheuvels, S., Dupret, M.A., Appourchaux, T., Scuflaire, R., Auvergne, M., Catala, C., Michel, E., Miglio, A., Montalban, J., Thoul, A., Talon, S., Baglin, A., Noels, A.: Science 324, 1540 (2009a)

Belkacem, K., Samadi, R., Goupil, M.J., Dupret, M.A., Brun, A.S., Baudin, F.: Astron. Astrophys. 494, 191 (2009b)

Belkacem, K., Dupret, M.A., Noels, A.: Astron. Astrophys. (2009c, in press). arXiv:0911.0908

Browning, M.K., Brun, A.S., Toomre, J.: Astrophys. J. 601, 512 (2004)

Chaplin, W.J., Houdek, G., Elsworth, Y., Gough, D.O., Isaak, G.R., New, R.: Mon. Not. R. Astron. Soc. 360, 859 (2005)

Dupret, M.A.: Bull. Soc. R. Sci. Liège 5-6, 249 (2002)

Dupret, M.A., Scuflaire, R., Noels, A., Thoul, A., Garrido, R., Moya, A., De Ridder, J., De Cat, P., Aerts, C.: Astrophys. Space Sci. 284, 129 (2003)

Dziemblowski, W.: Acta Astron. 27, 95 (1977)

Grigahcène, A., Dupret, M.A., Gabriel, M., Garrido, R., Scuflaire, R.: Astron. Astrophys. 434, 1055 (2005)

Lochard, J., Samadi, R., Goupil, M.J.: Astron. Astrophys. 438, 939 (2005)

Meakin, C.A., Arnett, D.: Astrophys. J. 667, 448 (2007)

Michel, E., Samadi, R., Baudin, F., Barban, C., Appourchaux, T., Auvergne, M.: Astron. Astrophys. 495, 979 (2009)

Musielak, Z.E., Rosner, R., Stein, R.F., Ulmschneider, P.: Astrophys. J. 423, 474 (1994)

Pesnell, W.D.: Astrophys. J. 363, 227 (1990)

Samadi, R.: In: Rozelot, J.P., Neiner, C. (eds.) Lecture Notes in Physics. Springer, Berlin (2010, in press)

Samadi, R., Goupil, M.: Astron. Astrophys. 370, 136 (2001) (SG01)

Samadi, R., Nordlund, Å., Stein, R.F., Goupil, M.J., Roxburgh, I.: Astron. Astrophys. 404, 1129 (2003)

Samadi, R., Nordlund, ̊., Stein, R.F., Goupil, M.J., Roxburgh, I.: Astron. Astrophys. 403, 303 (2003)

Samadi, R., Belkacem, K., Goupil, M.J., Dupret, M.A., Kupka, F.: Astron. Astrophys. 489, 291 (2008)

Scuflaire, R., Théado, S., Montalbán, J., Miglio, A., Bourge, P.O., Godart, M., Thoul, A., Noels, A.: Astrophys. Space Sci. 316, 83 (2008)

Scuflaire, R., Montalbán, J., Théado, S., Bourge, P.O., Miglio, A., Godart, M., Thoul, A., Noels, A.: Astrophys. Space Sci. 316, 149 (2008) 